\title{
In memoriam Bruno Durrer (1953-2016)
}

Bruno Durrer ist 2001 als Vertreter der Schweizerischen Gesellschaft für Gebirgsmedizin (SGGM) in die noch sehr junge Plattform Rettungswesen der FMH eingetreten. Anlässlich seiner ersten Sitzungsteilnahme stellte er das Basiskurs-Curriculum für den Sommer- und Winterkurs der Gebirgsärzte vor, das mit 100 Stunden Ausbildungszeit eine sehr umfangreiche theoretische und vorwiegend praktische Einführung in die relevanten Aspekte der Gebirgsmedizin vermittelte. Der damals notierte Protokolleintrag der Plattformsitzung stellt klar, dass «die Anforderungen an einen Retter im Gebirge ungleich höher seien als die in der Notfallstation eines Spitals», was körperlich und mental sicher absolut ausser Frage stand und zusätzlich auch fachlich angesichts der seinerzeit noch weitgehend ungelösten Struktur- und Qualitätsprobleme unserer Notfallstationen in der Schweiz sehr glaubhaft erschien.

Bruno war aber nicht nur für den Theorieteil der Gebirgsmedizin zuständig. Seine grosse praktische Erfahrung durch seinen unermüdlichen Einsatz als langjähriger Notarzt der Air-Glacier-Helikopterstation Lauterbrunnen, Höhlenretter und Bergführer war nicht nur im Lauterbrunnental bekannt. Er war ein geschätzter Ausbildner, Referent und Organisator von Symposien und Kursen in der Bergrettung, darüber hinaus viele Jahre lang in der medizinischen Ausbildung der Bergführerkurse des Schweizer Bergführerverbandes (SBV) aktiv - ein Engagement, das 2010 mit dem «King Albert Mountain Award» auch internationale Anerkennung fand.

\section{Er war ein geschätzter Ausbildner, Referent und Organisator von Symposien und Kursen in der Bergrettung.}

Aber Bruno war auch Hausarzt mit Leib und Seele, domiziliert in einer grossen Allgemeinpraxis in Lauterbrunnen. Deshalb war es nur folgerichtig, dass er im gleichen Jahr in der Plattform Rettungswesen der FMH neben seiner Arbeit für die SGGM auch noch das Mandat der SGAM übernahm, wofür ihn sein Vorgänger Kaspar Lüthi vorgeschlagen hatte. In dieser Funktion spielte er eine wichtige Rolle bei der Entwicklung unseres Leitbildes zum Rettungswesen in der Schweiz und den anderen im Lauf der Jahre entstandenen Publikationen. Dass die Rettungskette in der Schweiz massgeblich auf die Integration der medizinischen Grundversorger aufbaut und Notfallmedizin ein wichtiger Bestandteil der allgemeinmedizinischen Aus- und Weiterbildung bleibt, ist Bruno stets ein Herzensanliegen gewesen.

\section{Sein Engagement fand 2010 mit dem «King} Albert Mountain Award" auch internationale

\section{Anerkennung.}

Neben seiner Liebe zu den Bergen war Bruno Durrer auch ein begeisterter Taucher. Nach einem Tauchgang während seiner Ferien in Indonesien fühlte Bruno sich nicht gut und verstarb am 5. Dezember kurze Zeit später auf dem Weg ins Spital. Wir sind tief bestürzt und können uns die Plattform Rettungswesen ohne ihn nicht wirklich vorstellen. Unser Mitgefühl ist bei seinen Angehörigen, aber wir sind dankbar für die Zeit, die wir mit ihm verbringen durften.

Im Namen der FMH-Plattform Rettungswesen: Prof. Wolfgang Ummenhofer, Präsident; Dr. Remo Osterwalder, Vizepräsident FMH; Dr. Patrick Siebenfund, Präsident elect

Im Namen der Schweizerischen Gesellschaft für Notfallund Rettungsmedizin (SGNOR/SSMUS):

Dr. Barbara Schild, Co-Präsidentin; Prof. Heinz Zimmermann, Co-Präsident; Gabriela Kaufmann-Hostettler, Geschäftsführerin 